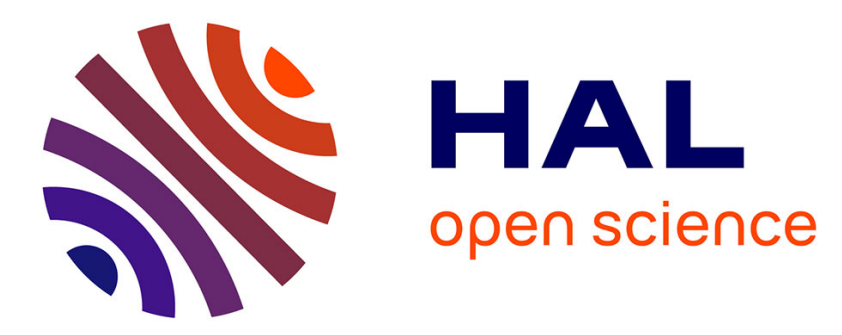

\title{
Oui mais elle est où la coupure là? Quand syntaxe et prosodie s'entraident ou se complètent
}

Anne Lacheret, Sylvain Kahane, Paola Pietrandrea, Mathieu Avanzi, Bernard Victorri

\section{- To cite this version:}

Anne Lacheret, Sylvain Kahane, Paola Pietrandrea, Mathieu Avanzi, Bernard Victorri. Oui mais elle est où la coupure là? Quand syntaxe et prosodie s'entraident ou se complètent. Langue française, 2011, 170, pp.61-80. halshs-00666668

\section{HAL Id: halshs-00666668 \\ https://shs.hal.science/halshs-00666668}

Submitted on 6 Feb 2012

HAL is a multi-disciplinary open access archive for the deposit and dissemination of scientific research documents, whether they are published or not. The documents may come from teaching and research institutions in France or abroad, or from public or private research centers.
L'archive ouverte pluridisciplinaire HAL, est destinée au dépôt et à la diffusion de documents scientifiques de niveau recherche, publiés ou non, émanant des établissements d'enseignement et de recherche français ou étrangers, des laboratoires publics ou privés. 


\title{
Oui mais elle est où la coupure, là ? Quand syntaxe et prosodie s'entraident ou se complètent
}

\author{
Anne Lacheret-Dujour (U. Paris Ouest, Modyco) \\ Sylvain Kahane (U. Paris Ouest, Modyco) \\ Paola Pietrandrea (U. Roma TRE / Lattice / Modyco) \\ Mathieu Avanzi (U. de Neuchâtel / U. Paris Ouest, Modyco) \\ Bernard Victorri (CNRS, Lattice)
}

\section{Introduction}

Un problème central dans l'étude de la compréhension des énoncés oraux est de déterminer le rôle que jouent les indices intonosyntaxiques dans la segmentation du continuum sonore en unités discursives et dans la gestion du flux informationnel (BlancheBenveniste et al. 1990, Charolles et al. 2002, Apothéloz et al. 2007, Mettouchi et al. 2007). Se posent notamment les questions suivantes : quel est le degré de congruence entre les différentes unités manipulées par la syntaxe et la prosodie, mais aussi de quelle manière prosodie et syntaxe s'éclairent réciproquement et collaborent à la construction du sens en contexte?

Aborder concrètement ces questions nécessite le développement de corpus doublement annotés prosodiquement d'une part, syntaxiquement d'autre part. Dans un premier temps, indépendantes l'une de l'autre, ces annotations sont ensuite mises en regard de manière à comprendre cet éclairage réciproque et aboutir au final à une annotation stabilisée, clairement explicitée et donc a priori reproductible sur des gros volumes de données.

L'objectif de l'article est d'illustrer les travaux conduits dans le cadre de l'ANR Rhapsodie (http://rhapsodie.risc.cnrs.fr/fr/index.html) en termes d'annotation prosodique d'une part, d'annotation syntaxique d'autre part à partir d'une analyse exhaustive de l'extrait CFPP2000 qui nous a été proposé. Nous présenterons les principes de nos segmentations prosodiques et syntaxiques, puis, nous verrons quelles corrélations existent entre nos unités prosodiques et syntaxiques. Nous formulerons des hypothèses sur les motivations syntaxiques du découpage prosodique et nous justifierons sous l'angle syntaxique les réajustements envisagés dans la segmentation prosodique. De la même façon, nous étudierons certaines décisions syntaxiques revisitées par la prosodie.

\section{Le traitement prosodique du corpus}

Notre recherche s'inscrit dans la continuité des réflexions menées sur l'interface prosodie-discours (pour une revue récente, voir les Symposiums internationaux organisés ces dernières années sur cette question), qui s'interrogent sur le rôle de la prosodie dans la segmentation du discours en unités fonctionnelles (fonction communicative et fonction de représentation). L'approche des faits prosodiques est ici inductive puisqu'elle se fonde au départ uniquement sur l'analyse des paramètres acoustiques et de leurs corrélats perceptifs pour faire émerger les unités prosodiques sans préjuger des fonctions auxquelles elles peuvent répondre. Cette question de la fonction/ou des fonctions ne pouvant être traitée que dans un second temps en mettant en regard les résultats issus du traitement prosodique avec les résultats fournis par une analyse syntaxique conduite indépendamment.

La structure prosodique manipulée s'articule autour de deux niveaux de segmentation : la segmentation du discours en périodes d'abord, l'organisation prosodique interne des périodes ensuite. Concernant cette dernière, nous retrouvons de façon classique la notion de groupe accentuel (GA) : dans un premier temps, chaque période est segmentable en une succession de GA sur les bases d'indices phonétiques, la question étant de savoir dans un second temps 
le type de relation que peuvent entretenir deux GA contigus dans une période : 1) relation de successivité et autonomie des GA ou 2) application d'un principe de dominance intonative, i.e. relation de dépendance marquée par un mécanisme d'emboîtement intonatif spécifique. Nous parlerons alors de processus d'empaquetages des GA ou encore de la formation de paquets intonatifs $(\mathrm{PI})^{1}$.

\subsection{Les périodes intonatives comme objet de discours}

Selon l'hypothèse qui fonde le concept de période (intonative), 1) le discours s'articule autour d'une succession de séquences, ou périodes qui viennent scander ce dernier en fonction de contraintes propres à sa genèse et à son organisation thématique; 2) les ruptures prosodiques fortes que l'on trouve dans un discours donnent à voir cette organisation (Lacheret et al. 2007, 2011). Autrement dit, ce premier niveau de découpage répond essentiellement à une contrainte discursive, les deux autres (identification des GA et des PI) seraient sous-tendus par des facteurs syntactico-sémantiques. Chaque période est donc vue comme une étape dans la structuration globale du discours et peut être typée en conséquence ; principalement ici : séquence d'ouverture, illustration, clôture, argumentation, explication, ou encore marqueur de discours rapporté ${ }^{2}$. Soit à titre d'illustration, la segmentation périodique suivante pour le début du corpus d'étude :

1. Séquence d'ouverture bon je reviens sur cette euh ce problème qui est un problème euh voilà de d'être chez moi combien de fois ça m'est arrivé

2. Séquence illustrative 1. bon ben là tu vas boulevard voltaire c'est pas loin euh tu tu j'y vais à pieds je suis chez moi je me conditionne dans mon appartement en me disant j'y vais à pieds moi ma voiture elle est garée dans la rue j'ai un stationnement résident

3. Séquence illustrative 2 (changement de séquence car déplacement spatial du locuteur intérieur > extérieur). je passe devant je ne peux pas m'empêcher d'ouvrir euh la porte de monter dedans et et d'aller euh à euh voilà cinq minutes en voiture ce qui me mettrait peut-être euh un petit quart d'heure à pieds

4. Séquence de clôture donc au dernier moment je prends ma voiture

Tableau 1. Segmentation du discours en périodes : illustration

Le découpage en périodes a été effectué de façon automatique avec le logiciel Analor (http://www.lattice.cnrs.fr/Analor.html) et vérifié manuellement par deux des auteurs. Nous ne reviendrons pas sur les détails de l'algorithme largement présentés par ailleurs (Lacheret \& Victorri 2002, Avanzi et al. 2008), nous limitant ici à ses fondements majeurs. En premier lieu, le marquage d'une frontière périodique marquée par l'interruption du flux verbal passe également par l'activation simultanée d'un certain nombre de paramètres mélodiques et temporels, la pause étant un indice de segmentation nécessaire mais non suffisant. En conséquence, les critères retenus sont de trois ordres: la durée d'une pause détectée, l'amplitude d'un contour repéré comme potentiellement terminal de période, l'amplitude d'un saut mélodique marqueur de réinitialisation mélodique, i.e. la différence de hauteur entre la dernière valeur de $\mathrm{F} 0$ précédant la pause et la première valeur de $\mathrm{F} 0$ suivant la pause. A cela s'ajoute la nécessaire absence d'un euh contigu à la pause. En second lieu, pour proposer une explication cognitive de cette organisation, on pose deux principes sous-jacents au processus de segmentation: un principe de quantité et un principe de compensation. Selon le premier, il faut que l'activation d'un paramètre atteigne un certain seuil pour que son rôle soit considéré comme pertinent dans la coupure en période. Selon le second, l'absence de

\footnotetext{
${ }^{1}$ Toute chose égale par ailleurs, une équivalence conceptuelle est envisageable avec les deux unités centrales de la phonologie prosodique : le syntagme phonologique et le syntagme intonatif, où plus largement les domaines majeur et mineur postulés par les différents courants qui se réclament de cette approche aujourd'hui (Avanzi 2011). Une analogie plus immédiate pourra être faite avec les travaux pionniers de P. Mertens (1987).

${ }^{2}$ Cette annotation n'est pour l'heure qu'exploratoire, inspirée des travaux sur le discours écrit (Adam 1999). Elle demande à être approfondie et stabilisée au regard des travaux sur l'oral.
} 
variation significative d'un des 3 paramètres peut être compensée par l'activation forte d'un autre.

\subsection{L'identification des proéminences et de leurs degrés à l'intérieur des périodes}

Sous l'angle perceptif, l'identification des proéminences syllabiques ${ }^{3}$ repose sur l'application d'un principe de contraste (figure/fond), une syllabe se détachant dans le flux verbal de son entourage immédiat et contrastant ainsi avec son environnement. Ce contraste est plus ou moins net selon les contextes, ce qui revient à établir des degrés de proéminence. Pour l'heure, nous proposons d'en distinguer deux : proéminence moyenne vs. proéminence forte. On peut supposer qu'à ces degrés sont associés des corrélats acoustiques précis, un tel traitement est donc implémentable. Nous posons ensuite l'hypothèse que ces différences de degrés donnent à voir la structuration syntactico-sémantique du discours, la robustesse de l'annotation prosodique envisagée est donc à ce niveau cruciale pour l'analyse linguistique du corpus. De la même manière que pour les périodes, l'identification automatique des degrés de saillance prosodique, validée manuellement par deux des auteurs, repose sur un principe de quantité. Quatre variables sont considérées pour lesquelles les seuils ont été fixés manuellement sur les bases de l'expertise phonétique proposée dans la littérature (pour une présentation détaillée, voir Avanzi et al. 2011) : 1) rehaussement mélodique de la syllabe, 2) allongement syllabique, 3) amplitude du contour mélodique associé à la proéminence, 4) prise en compte de la pause silencieuse quand elle est présente dans le contexte droit.

La distribution des proéminences étant variable ( \pm finales de mots), chaque proéminence terminale est ici considérée comme démarcative, i.e. identification de la fin d'un GA. A ce niveau de l'analyse donc, les périodes sont segmentées en une succession de GA.

Ainsi pour la première de nos quatre périodes initiales, le traitement des proéminences donne les sorties suivantes :

$$
\begin{aligned}
& \text { bon je reviens } \mathrm{p} \text { sur cette euh ce problème } \mathrm{p} \text { qui est un problème } \mathrm{P} \\
& \text { euh voilà de d'être chez moi } \mathrm{P} \text { combien de fois ça m'est arrivé } \mathrm{P}
\end{aligned}
$$

\subsection{Des groupes accentuels aux paquets intonatifs}

Pour continuer le traitement, nous posons le principe suivant: pour toute syllabe terminale, l'identification d'une proéminence forte conduit à l'inclusion du ou des groupe(s) accentuel(s) qui précède(nt), quel que soit leur nombre, dans un groupement intonatif plus large que nous appelons paquet intonatif (PI). La mise en place de la structure prosodique interne à la période repose donc sur l'application de principes de liage ou au contraire de rupture entre deux GA contigus.

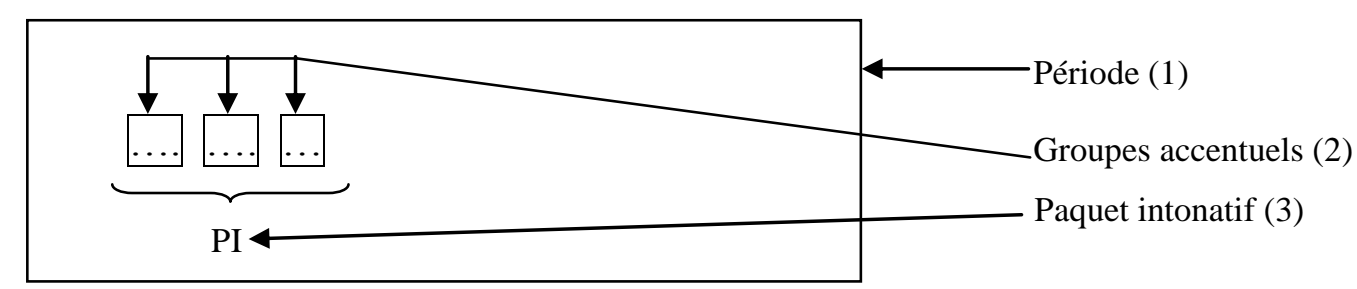

Tableau 2. Les différentes unités prosodiques telles qu'elles sont successivement identifiées : 1) découpage en périodes, 2) segmentation d'une période en GA, 3) regroupement des GA en PI.

\footnotetext{
${ }^{3}$ La terminologie employée (proéminence et non accent) illustre le parti pris de la démarche résolument émergente, écartant tout a priori fonctionnel (qui dit accent dit fonction accentuelle).
} 
En sortie du traitement, nous obtenons notre degré de segmentation intermédiaire entre la période et le GA : le PI, soit pour la période 1, 3 PI :

(2)

\begin{tabular}{|l|l|l|}
\hline PI1 & bon je reviens & sur cette euh ce problème qui est un problème \\
PI2 & euh ${ }^{4}$ voilà de d'être chez moi \\
PI3 & combien de fois ça m'est arrivé \\
\hline
\end{tabular}

\section{Le traitement syntaxique du corpus}

Nous nous inscrivons dans un cadre classique qui distingue deux modules, à savoir la micro- et la macrosyntaxe (Blanche-Benveniste et al. 1990, Berrendonner 1990, Cresti 2000, Andersen \& Nølke 2000) en leur donnant une indépendance assez forte. L'objectif majeur : tenter d'une part de mieux délimiter ce que l'on aimerait appeler une unité syntaxique maximale et, d'autre part, aborder les cas difficiles sur ce corpus et la manière dont nous les avons résolus. Nous considérons deux unités d'analyse centrales, l'unité rectionnelle (UR) et l'unité illocutoire (UI), qui sont respectivement les unités principales de la micro- et de la macrosyntaxe (Benzitoun et al. 2010).

\subsection{Les unités rectionnelles (UR)}

L'UR, unité maximale de la microsyntaxe, est une séquence construite autour d'une tête qui ne dépend microsyntaxiquement d'aucune autre unité. De cette tête dépend un ensemble d'éléments reliés par des relations de rection ou d'entassement paradigmatique. Un élément est considéré comme régi lorsqu'il se voit imposer certaines contraintes (partie du discours, place linéaire, marqueur morphosyntaxique). Dans l'extrait suivant, on trouve 5 UR. La sémantique des délimiteurs <, > et // sera explicité dans la section suivante.

(3) L1 : le deux-roues < ça vous déplairait pas //

L2 : le vélo < si > pour essayer une fois //

Nous considérons, à la suite des travaux de l'école d'Aix, qu'il y a entassement lorsque plusieurs syntagmes viennent occuper la même position régie. L'entassement de $\mathrm{X}$ et $\mathrm{Y}$ est noté $\{\mathrm{X} \mid \mathrm{Y}\}$. Cette notion recouvre aussi bien des cas de disfluence (2)a-b que de coordination $(2) c-d$ :

(4) a. "euh" $\{\mathrm{tu}|\mathrm{tu}| \mathrm{j}$ '\} y vais à pieds //

b. "euh" \{ y a quand même $\mid$ y a $\}$ \{des $\mid$ des $\}$ sous sur le compte) //

c. je n'peux pas m'empêcher $\{$ d'ouvrir "euh" la porte $\mid$ de monter dedans $\mid \wedge$ et d'aller

"euh" à "euh" "voilà" cinq minutes en voiture \} //

d. par contre < j'ai essayé le vélib \{deux | trois \} fois en mettant ma carte bleue //

\subsection{Les unités illocutoires (UI)}

L'UI est une portion de discours qui réalise un seul acte illocutoire, c'est-à-dire une assertion, une interrogation ou une injonction. Une UI se décompose en composantes illocutoires (CI), dont l'une d'elles, le noyau, porte la force illocutoire. Le noyau d'une UI (comme par exemple le segment je l'sais en (3) est autonomisable, c'est-à-dire qu'il peut être découpé et reconnu comme un énoncé syntaxiquement et prosodiquement bien formé sans changement de sens (Martin 2009). Il est susceptible de porter des modalités et de rentrer dans la portée d'une interrogation (Blanche-Beneveniste et al 1990). Il peut être pris en charge par l'interlocuteur qui peut le questionner ou le réfuter. Les ad-noyaux, c'est-à-dire les CI non autonomisables et rattachées ainsi au noyau, sont séparés du noyau par les balises < et >,

\footnotetext{
${ }^{4}$ Où par défaut et dans l'attente d'une analyse approfondie, les marqueurs « euh » sont associés au PI de droite.
} 
selon qu'il s'agit de pré-noyaux (ad-noyaux précédant le noyau) ou de post-noyaux (adnoyaux postposés au noyau). La fin d'une UI est indiquée par //. Le symbole \& note une incomplétude syntaxique :

(5) je l'sais > qu'j'suis perdante //

(6) "non" ^mais j'crois que je suis \& // comme j'ai jamais fait quasi d'vélo d'ma vie < "euh" $\{$ en tant que vraie parisienne $\mid \wedge$ et en étant très peu partie à la campagne $\}<\mathrm{j}$ 'suis $\{$ pas $\mid$ pas $\}$ très à l'aise $/ /{ }^{\wedge}$ mais pour faire les petites distances $<\& / /$

En général, les CI sont des UR, mais la rection et l'entassement peuvent aller au delà de la CI ou même de l'UI (Benzitoun et al. 2010, Deulofeu et al. 2010). Lorsqu'une balise illocutoire $(<,>$ ou //) n'est pas une frontière d'UR, nous ajoutons un + :

(7) a. ${ }^{\wedge}$ donc au dernier moment $<+$ je prends ma voiture //

b. ${ }^{\wedge}$ et alors < comment vous vivez $\{$ les $\mid$ la $\mid$ la $\}$ politique de la ville de Paris //+ qui est quand même une chasse aux voitures //

La proposition relative en (5)b est bien une UI : ce segment n'est pas dans la portée de l'interrogation qui précède et forme une assertion séparée, qui peut être prise en charge par l'interlocuteur et être réfutée (par c'est pas vrai) et dont on peut modifier la force illocutoire. Le fait qu'il s'agit d'une relative ne permet pas d'en faire réellement une interrogative, mais on peut lui adjoindre une tag question : qui est quand même une chasse aux voitures, vous trouvez pas?

\subsection{Insertion et enchâssement d'UI}

Les UI ne sont pas toujours à la suite l'une de l'autre. Deux complications peuvent se présenter. La première est l'insertion d'une UI au milieu d'une autre. Une telle UI est placée entre (...) et terminée par // (cf. c'est une vraie question hein en (6)). Nous utilisons également les (...) pour indiquer l'in-noyau, c'est-à-dire un élément qui comme un pré- ou un post-noyau ne possède pas de force illocutoire propre et vient s'insérer au milieu du noyau : (cf. en termes de temps en (6)).

(8) ^mais \{ pour l'instant (c'est une vraie question "hein" //) | pour l'instant \} < c'est quand même (en termes de temps) rentable aussi d'prendre la voiture //

L'autre complication est fournie par le discours rapporté : il s'agit dans ce cas d'une UI ou d'une séquence d'UI qui sont régies par un verbe de discours. Ces UI sont enchâssées : tout en étant de vraie UI possédant leur propre force illocutoire, elles appartiennent aussi à l'UR et l'UI du verbe recteur du discours rapporté. Nous les indiquons entre [...], chaque UI enchâssée étant terminée par un //.

(9) a. je m'conditionne dans mon appartement en m'disant [j'y vais à pieds //] //

b. sur le coup $<$ j'me dis [ j'vais mettre cinq minutes //] //

Il se peut qu'un discours rapporté ne soit pas enchâssé et régi par un verbe de discours. Notre corpus contient un tel cas lorsque l'interviewée rapporte un dialogue avec ses filles, où réponse des filles, qui constitue la deuxième partie de (10), ne comporte aucune marque syntaxique permettant de l'identifier comme un discours rapporté. Seules la prosodie et la sémantique permettent ici d'en juger et nous ne notons rien en syntaxe.

(10) ^puisque moi < "voilà" j'peux m'dire [^mais j'viens t'chercher // tu finis tard // tu seras $\&$ voilà //] // non non non // laisse-moi marcher //= laisse-moi marcher // ^donc "euh" non non //

\subsection{Introducteurs d'UI et UI associées}


Nous notons encore deux types d'éléments dont nous pensons qu'ils présentent des particularités intonosyntaxiques. Les premiers sont les introducteurs d'UI, que nous indiquons en les faisant précéder d'un ^. Il s'agit d'éléments qui possèdent une position initiale fixe, fonctionnent comme connecteurs avec l'UI qui précède et sont généralement prosodiquement intégrés au segment qui suit, qu'il s'agisse d'un pré-noyau ou du noyau. Nous utilisons le même symbole pour noter les conjonctions de coordination dans les entassements, car il s'agit souvent des mêmes éléments lexicaux et que leurs propriétés sont comparables. Nous renvoyons aux exemples qui précèdent.

Les deuxièmes éléments que nous isolons sont certains marqueurs de discours, que nous notons entre "..." :

(11) a. "bon ben" là < tu vas boulevard Voltaire //

b. "ah ben" $\{\mathrm{je} \mid \mathrm{je}\}$ suis pour //

Ces éléments ne sont pas régis et forment donc des CI séparés. Nous pensons qu'ils doivent être distingués des noyaux et des ad-noyaux. Bien qu'ils ne forment pas des UI en tant que tels, ils peuvent potentiellement être utilisés de manière autonome. Nous les appelons des unités illocutoires associées (UIA) (Kahane \& Pietrandrea, à paraître) et nous reviendrons dessus lorsque nous évoquerons le problème posé par les mots-phrases.

\section{Analyse intonosyntaxique}

Nous présentons successivement les tendances générales pour les corrélations observées entre les deux niveaux d'annotation, puis nous examinons sur des cas précis l'éclairage réciproque de la prosodie et de la syntaxe.

\subsection{Corrélations entre prosodie et syntaxe}

Le premier point à noter est qu'il y a une corrélation assez forte entre frontières prosodiques et frontière syntaxiques ${ }^{5}$ :

- 48 des 72 frontières de PI sont des frontières d'UI (soit 65\%). Parmi les 25 frontières de PI restantes, 9 tombent entre un noyau et un ad-noyau, 3 sont suivies d'un euh et 3 figurent entre deux termes d'une liste paradigmatique. Restent 6 frontières de PI (soit $8 \%$ ) non motivées syntaxiquement, par exemple :

(12) PI1 en rentrant

PI2 dans un processus

PI3 que j'n'aime

PI4 pas trop vis-à-vis d'moi //

PI5 c'est-à-dire que "euh" \{il faut

PI6 me mettre des li- $\mid$ il faut quand même me mettre des limites $\}$ pour m'empêcher de prendre la voiture // alors que ça devrait venir de moi //

- 47 des 54 frontières d'UI sont des frontières de PI (soit 87\%). Les 5 frontières restantes pourraient correspondre à des constructions particulières, comme cet enchaînement de 4 UI en un PI qui font montre d'un fort parallélisme syntaxique et sémantique (que nous notons par un //=)

\footnotetext{
${ }^{5}$ En cas de comparaison avec les autres articles portant sur ce corpus, rappelons que les chiffres qui suivent concernent uniquement la partie de l'interviewée, hors chevauchements. Les décomptes prennent en compte les décisions commentées dans les sections suivantes.
} 
(13)

pareil qu'elles marchent $/ /=$ elles prennent pas l'métro pour faire quatre cinq stations $/ /=$ elles prennent pas l'métro $/ /=$ elles y vont à pieds $/ /$

- Des 16 frontières entre noyau et ad-noyau, 9 sont des frontières de PI et 6 de GA. Reste un GA surprenant contenant à la fois une frontière de pré-noyau et une frontière de début d'UI enchâssée :

\section{^puisque moi < "voilà" j'peux m'dire [^mais j'viens t'chercher // tu finis tard // ]//}

- Seules 3 des 5 frontières d'UI enchâssées sont des frontières de PI.

Les frontières de période sont obligatoirement des frontières d'UI et les périodes regroupent entre 1 et 6 UI. Nous n'avons pas étudié en détail les éventuelles propriétés syntaxiques de ces regroupements.

\subsection{L'éclairage de la prosodie par la syntaxe}

L'expertise syntaxique nous permet de valider partiellement le découpage prosodique proposé, en suggérant dans l'immédiat des reconfigurations fonctionnellement contraintes (différence entre accents démarcatifs et focaux notamment) et en montrant les problèmes que cela pose, mais également en épinglant certaines structures spécifiques liées au travail de formulation ou à la rhétorique discursive qui mettent en lumière la nécessité d'enrichir le modèle prosodique, celui-ci ne pouvant se réduire à l'application d'un seul et unique principe de dominance intonative.

\subsubsection{Le découpage périodique et la formation des constituants internes à la période}

Le découpage en périodes sur des bases strictement phonétiques rend compte d'un mode de segmentation régulier reposant clairement sur une logique d'organisation thématique $\mathrm{du}$ discours. Cette organisation a des corolaires syntaxiques, nous l'avons vu : une fin de période correspond nécessairement à une fin d'unité illocutoire (UI), i.e. une UI ne peut pas être à cheval sur deux périodes, les relations entre frontières prosodiques (PI) et composantes illocutoires à l'intérieur de la période sont dans l'ensemble homogènes, etc. Le statut des GA est en revanche moins clair et parmi les décisions à prendre en définitive, certaines questions restent posées. Pour mémoire, la structuration interne des périodes sur les bases de l'identification perceptive des proéminences de mots et de leurs corrélats acoustiques fournis par le calcul automatique nous a conduits à définir la règle suivante : toute proéminence terminale constitue un indice de segmentation interne et permet de repérer la fin d'un GA ; en pratique, deux problèmes majeurs demeurent. Premièrement, il semble difficile d'envisager une coupure à l'intérieur d'un groupe syntaxiquement très cohésif (j'vais mettre cinq) (minutes), (non non elles adorent) (marcher) et ce d'autant plus que la proéminence tombe sur un mot appartenant à la classe des clitiques si on se réfère aux grammaires d'usage (je) ( $m$ ' conditionne) (Riegel et al. 1993). Nous avons donc considéré qu'il s'agissait là de proéminences focales et fusionné les GA. Ensuite, quel sort prosodique réserver en termes de découpage accentuel aux contextes d'hésitation? Dans l'exemple qui suit, le découpage que nous avons retenu après réajustement syntaxique est-il en définitive le plus pertinent? (faudrait que j' \&) (c habitude). A ce stade du travail, la question reste posée.

\subsubsection{Questions en suspens : scansions et travail de formulation, structure de liste et entassements}

Nous avons proposé d'interpréter de façon univoque le non regroupement de deux GA contigus en paquets intonatifs comme des indices de rupture syntactico-sémantiques $(§ 1.2)$. 
Quelles sont les limites de cette interprétation quand il s'agit d'analyser de la parole spontanée ? Comment élargir le processus interprétatif ? En quoi l'expertise syntaxique peutelle nous aider dans cette démarche ?

Dans la parole spontanée, où le processus de structuration du message est simultané à sa planification, il existe des espaces de scansion au sein desquelles les unités prosodiques sont en quelque sorte posées à plat les unes à la suite des autres sans effort de hiérarchisation, i.e. de marquage explicite de dépendance des unités, le locuteur étant sans doute trop pris par ailleurs dans son travail de formulation. Ainsi, dans l'exemple (12), l'éclatement de noyau d'UI en plusieurs PI met en lumière les limites de l'unicité interprétative: les ruptures prosodiques ne sont pas toujours les marqueurs de ruptures syntactico-sémantiques.

Echappent également au processus d'empaquetage intonatif certaines constructions symétriques qui se font échos et s'enchaînent jusqu'à un point de clôture prosodiquement marqué. Dans la période 6 (exemple (15)), ce principe est actualisé par la réitération d'un profil intonatif unique sur les 3 expansions nominales (1) de m'garer, (2) de tourner, (3) de faire des ronds: déclinaison mélodique et réinitialisation intonative sur chaque groupe (geste global descendant, marquage régulier gauche-droite de chaque expansion nominale répétée (2 et 3) par une proéminence syllabique initiale et finale de séquence).

(15) PI1 $\wedge$ mais

PI2 le temps \{ de m'garer |

PI3 de tourner|

PI4 de faire des ronds

PI5 pour pas mal me garer et tout $\}<$

PI6 je sais qu' je suis perdante //

Là encore, l'analyse syntactico-sémantique non seulement explique ces constructions prosodiques mais les appelle irrévocablement. Autrement dit, l'organisation prosodique ici rencontrée peut être considérée comme la prosodie typique des constructions par entassement épinglées par la syntaxe.

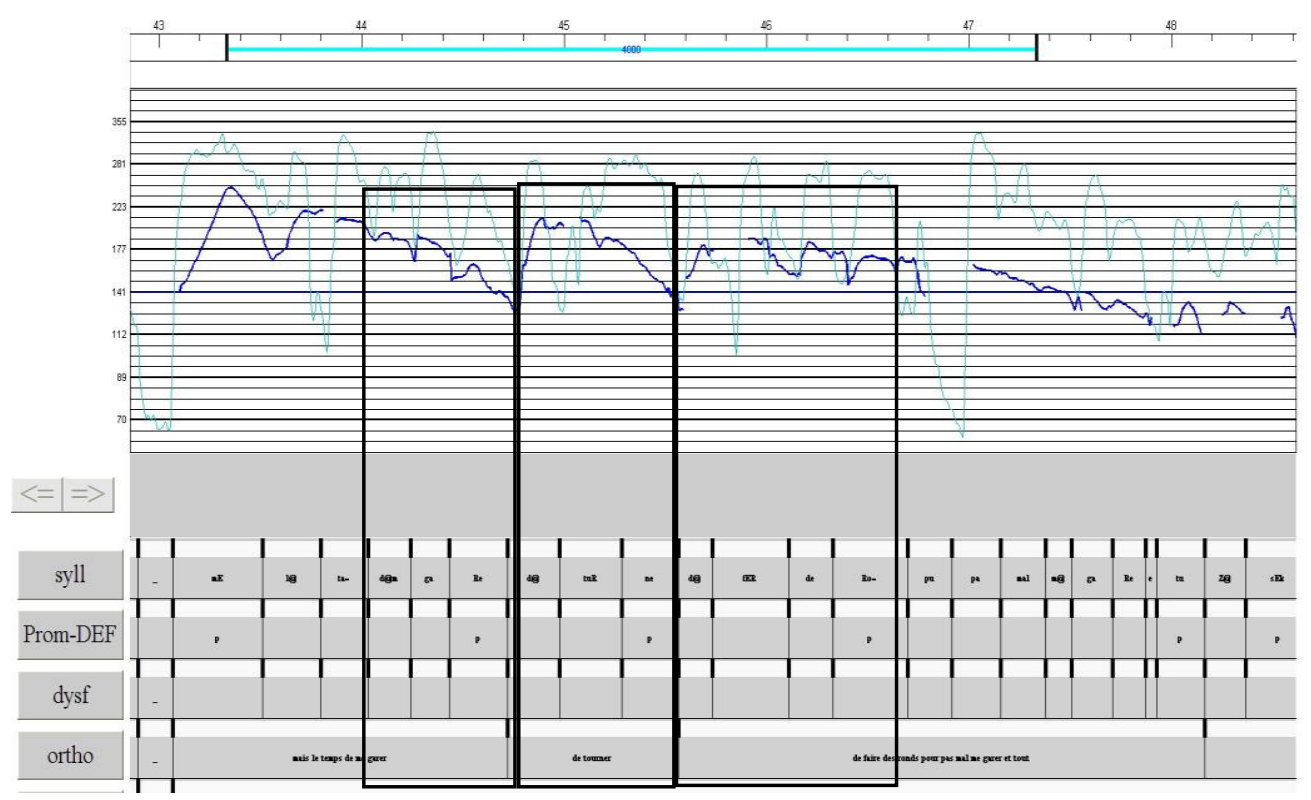

Figure 1. Profil prosodique de l'entassement en (15). 


\subsection{L'éclairage de la syntaxe par la prosodie}

Trois points sont présentés ici : le statut syntaxique du GN détaché à gauche dans des constructions topicalisées, les relations entre UR et UI, le statut ambigu de certaines unités appelées mots-phrases.

\subsubsection{Double marquage du sujet}

Il a été souvent noté, dans les productions orales, une forte proportion de double marquage du sujet, c'est-à-dire un GN précédent un pronom clitique sujet avec lequel il coréfère. Ceci amène certains auteurs à considérer que ce pronom clitique est en fait un affixe verbal (Miller \& Sag 1997, Culbertson \& Legendre 2008) et que le GN est bien le sujet. Si tel est le cas, ce GN devrait donc appartenir au noyau et non pas former un prénoyau. Dans notre corpus, nous avons un cas de double marquage où le groupe nominal coréférent au pronom clitique sujet est intégré au GA du verbe (cf. Avanzi et al. (2010) pour une étude plus systématique de cette situation). Nous considérons donc que ce GN appartient au noyau

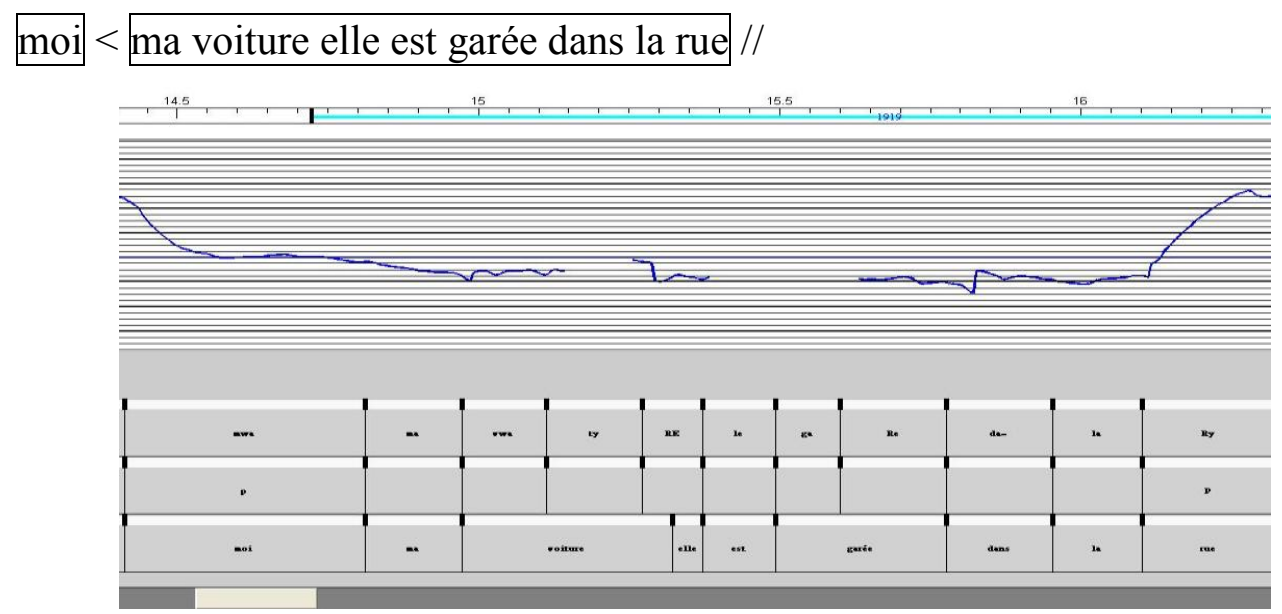

Figure 2. Profil mélodique de la séquence (16).

\subsubsection{Une UR en deux UI}

Notre corpus montre un cas d'une UR divisée en deux périodes et 5 PI. En cohérence avec le fait qu'une fin de période est nécessairement une fin d'UI, nous considérons qu'il s'agit donc d'une UR réalisée en deux UI. Une frontière d'UI qui n'est pas une frontière d'UR est indiquée par //+.

\begin{tabular}{|l|l|l|}
\hline P14 & PI5 & Aet elle m'est refusée $/ /+$ \\
\hline P15 & PI1 & même \\
& PI2 & si \\
& PI3 & "euh" $\{$ y a quand même $\mid$ y a $\}$ des $\mid$ des $\}$ sous sur le compte $/ /$ \\
& PI4 & Adonc "euh" voilà // \\
\hline
\end{tabular}

Considérer que cette UR forme deux UI revient à considérer qu'il s'agit de deux assertions, c'est-à-dire que la locutrice asserte deux choses : sa carte lui est refusée et elle lui est refusée même si il y a quand même des sous sur son compte. Il n'est pas surprenant que cette UR forme deux UI et elle pourrait même facilement former deux tours :

(18) L1 : et ma carte m'est refusée

L2 : même si tu as des sous sur le compte? 
Le corpus fournit d'ailleurs un exemple d'une UR sur deux tours de paroles, où la deuxième UI est un syntagme qui s'entasse sur une portion de la première UI :

(19) L2 : vos filles ont \{quel âge $\mid\} / /+$

L1 : ^alors $\{\mid$ dix-sept et demi et quinze ans $\} / /$

\subsubsection{UI ou non?}

Ce corpus de 3 minutes contient un grand nombre de «mots-phrases » (pour reprendre un terme introduit par Tesnière 1959) : 3 oui, 1 si, 12 non, 7 voilà. Ces éléments nous ont posé un problème d'analyse. Certains forment clairement le noyau d'une UI et peuvent même avoir prénoyau et/ou postnoyau :

(20) PI1 le vélo <

PI2 si > pour essayer une fois //

(21) PI1 "c'est bon" j'aurais pas toujours cette pensée sur d'autres domaines //

PI2 mais sur celle-là < oui //

Mais, dans d'autres cas, ces mots-phrases ne forment pas un GA, ce qui met en doute leur statut en tant qu'UI. Ainsi dans l'exemple (22), la première occurrence de non est une réponse à la question qui précède. Mais on a ensuite un GA qui contient deux amorces d'UI et une UI complète, ainsi que 4 occurrences de non. Il est très improbable qu'un GA contienne 7 UI, vu qu'il est déjà exceptionnel qu'un GA contienne deux UI (cf. (13) pour le seul autre exemple du corpus avec deux UI dans un GA). Nous pensons donc que ces occurrences ne fonctionnent pas comme de véritables noyaux, mais qu'il s'agit plutôt d'UI dont la force illocutoire est très atténuée et qui fonctionnent en combinaison avec une véritable UI. Nous les notons comme des UIA :

(22)

\begin{tabular}{|l|l|l|l|}
\hline L1 & & \multicolumn{2}{|c|}{ 'et c'est aussi des accros d'la voiture // } \\
\hline L2 & P18 & PI1 & "euh" non // \\
\hline & P19 & PI1 elles marchent //= \\
& & PI2 elles s' \& "non non" elles ont tendance à \& //= "non non" elles adorent marcher // \\
\hline
\end{tabular}

On peut également noter les séquences oui mais ou non mais dans lesquelles le motphrase est prosodiquement lié au mais au point de former un mot prosodique :

(23) "non" ^mais j'crois que je suis \& //

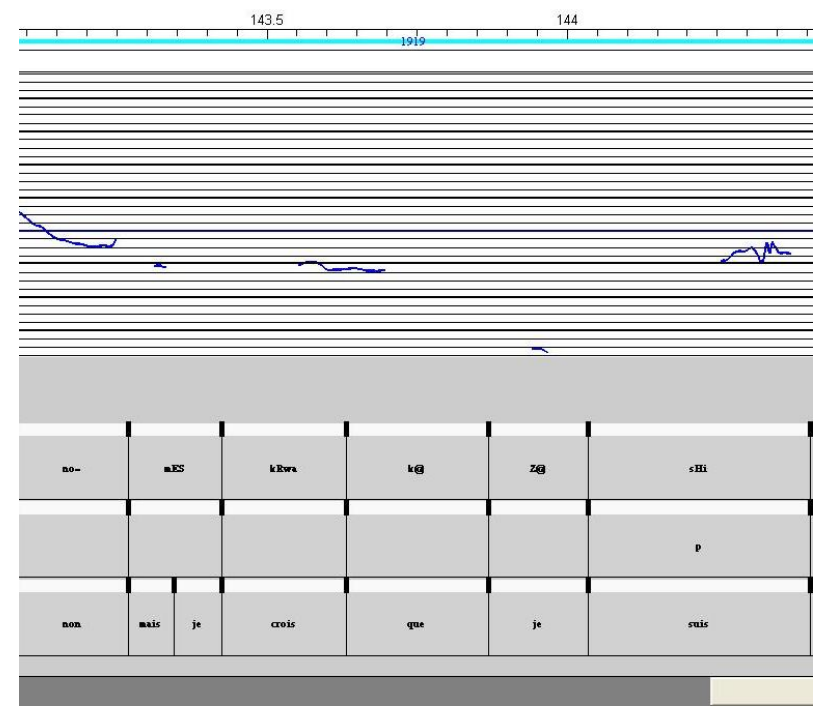


Figure 3. Profil mélodique de l'énoncé non mais je crois que je suis

De la même façon, nous traiterons voilà tantôt comme une UI, tantôt comme une UIA selon qu'il est ou non associé à une autre UI et forme ou non un GA distinct :

(24) PI1 oui //

PI2 y a un côté de facilité \{ d'passer devant sa voiture |

PI3 ^et de s'dire

PI4 "bon ben" j'la prends //] \} //

PI5 [\{et "euh"|^et \} voilà "quoi" //

PI1 ^puisque moi < "voilà" j'peux m'dire [^mais j'viens t'chercher //

Une analyse du rôle des mots-phrases lorsqu'ils ne forment pas des UI reste à faire. Il semble qu'ils n'ajoutent pas de contenu au savoir partagé (à la différence d'un oui ou d'un non qui répond à une question explicite et donc valide/invalide le contenu de cette question), mais jouent plutôt un rôle de commentaire.

\section{Conclusion}

En partant de l'observation d'un échantillon de parole spontanée extrait du CFPP2000, nous avons exposé la méthode développée dans le cadre de l'ANR Rhapsodie pour le traitement intonosyntaxique de corpus oraux. Le travail s'articule en deux temps : 1) une étape d'identification et d'étiquetage d'unités qui repose sur deux annotations conduites indépendamment l'une de l'autre, tant en ce qui concerne la nature des annotations (prosodiques vs syntaxiques) que les chercheurs qui annotent (deux équipes différentes travaillant indépendamment l'une de l'autre, 2) une étape d'analyse où prosodistes et syntacticiens se retrouvent pour observer et croiser les annotations obtenues afin de proposer en sortie une étude intonsyntaxique du corpus aussi cohérente et explicite que possible. L'objectif a donc été ici de 1) présenter la méthode d'annotation modulaire (modules de traitement prosodique et syntaxique autonomes), 2) illustrer quantitativement et qualitativement la lecture intonosyntaxique de données ainsi annotées, 3) épingler les cas qui résistent à l'analyse tant sous l'angle de la prosodie que de la syntaxe, les questions théoriques que cela pose et les pistes envisagées pour les résoudre.

\section{Références bibliographiques}

ADAM J.-M. (1999), Linguistique textuelle: des genres de discours au texte, Paris, Nathan.

ANDERSAN H. L \& NØLKE H. (éd.) (2002), Macro-syntaxe et macro-sémantique, Actes du colloque international d'Arhus, mai 2001, Bern, Peter Lang.

APOTHÉLOZ D., GROBET A. \& PEKAREK DOEHELER S. (éd.) (2007), Séquentialité et mouvements dans le discours, Cahiers de praxématique, 48.

AVANZI M., LACHERET A. \& VICTORRI B. (2008a), «Analor: un outil d'aide pour la modélisation de l'interface prosodie-grammaire », Travaux de linguistique du CERLICO, $n^{\circ} 21$ : Grammaire et prosodie, C. Colin (éd.), 27-46.

AVANZI M., LACHERET A., \& VICTORRI B. (2008b), "Analor, a tool for semi-automatic Annotation of French prosodic structure », Speech Prosody 2008, Campinas Bresil, http://www.sp2008.org/.

AVANZI M., GENDROT C. \& LACHERET A. (2010), « Is there a prosodic difference between leftdislocated and heavy subjects? Evidence from spontaneous French », Speech Prosody, Chicago, http://speechprosody2010.illinois.edu/.

AVANZI M. (2011), L'interface prosodie/syntaxe en français parlé. Dislocations, incises et asyndètes, Thèse de Doctorat, Université Neuchâtel/Paris Ouest Nanterre.

AVANZI M., LACHERET A., OBIN N. \& VICTORRI B. (2011 à par), "Vers une modélisation continue de la structure prosodique : le cas des proéminences accentuelles », Journal of French 
Language Studies 21.2 (Special Issue on French Prosody), M. Avanzi \& E. Delais-Roussarie (éd.).

BLANCHE-BENVENISTE C., BILGER M., ROUGET C. \& VAN DEN EYNDE K. (1990), Le français parlé : études grammaticales, Paris, Éditions du CNRS.

BENZITOUN C, DISTER A, GERDES K., KAHANE, S. PIETRANDERA P. \& SABIO F. (2010), «

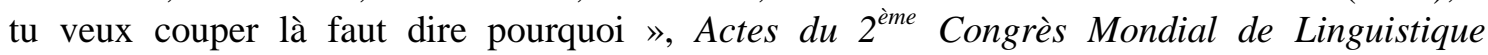
Française, La Nouvelle Orléans.

BENZITOUN C,, DEBAISIEUX J.-M., DEULOFEU J., GERDES K., KAHANE S. LEFEUVRE F., PIETRANDERA P., ROSSI-GENSANE N., SABIO F. \& VICTORRI B. (en construction), Guide d'annotation syntaxique Rhapsodie, http://rhapsodie.ilpga.fr/wiki.

BERREDONNER A. (1990), «Pour une macro-syntaxe », Travaux de Linguistique, 21: 25-36.

CHAROLLES M., LE GOFFIC P. \& MOREL M.-A. (éd.) (2000) Y a-t-il une syntaxe au-delà de la phrase, Verbum, XXIV, 1-2.

CRESTI E. (2000a), Corpus di italiano parlato, $\mathrm{Vol} \mathrm{I}^{\circ}$, $\mathrm{II}^{\circ}, \mathrm{CD}-\mathrm{ROM}$, Firenze, Accademia della Crusca.

CRESTI E. (2000b), « Critère illocutoire et articulation informationnelle », in M. Bilger (éd.), Corpus. Méthodologie et applications linguistique, Paris, Champion, 350-367.

CULBERTSON, J. \& LEGENDRE G. (2008), «Qu'en est-il des clitiques sujet en français oral contemporain ? ", Actes du $1^{\text {er }}$ Congrès Mondial de Linguistique Française, Paris.

DEULOFEU J., DUFFORT L., GERDES K, KAHANE S., PIETRANDREA P., (2010 « Depends on What the French Say Spoken Corpus Annotation With and Beyond Syntactic Functions.» Proceedings of the Fourth Linguistic Annotation Workshop (LAW IV), Uppsala, Suède.

LACHERET-DUJOUR A. \& VICTORRI B. (2002), « La période intonative comme unité d'analyse pour l'étude du français parlé : modélisation prosodique et enjeux linguistiques », Verbum, M. Charolles (éd.), 55-72.

LACHERET A \& VICTORRI B., AVANZI M. (2007), «La mise en scène intonative dans la description d'itinéraires en milieu urbain », Travaux neuchâtelois de linguistique, $n^{\circ} 47$ : Structuration grammaticale et structuration discursive, M. Avanzi \& A.S. Horlacher (éd.), 79102.

LACHERET A., AVANZI M. \& VICTORRI B. (2011), « Schématisation discursive et schématisation intonative : question de genre ? », Sciences du langage : carrefours et points de vue, R. Missire (éd.), Academia Bruylant.

MARTIN Ph. (2009), L'intonation du Français, Paris, Armand Colin.

MERTENS P. (1987), L'intonation du français : de la description linguistique à la reconnaissance automatique, Thèse de Doctorat, Université de Louvain.

METTOUCHI A., LACHERET-DUJOUR A., SILBER-VAROD V. \& IZRE'EL S. (2007), « Only Prosody? Perception of speech segmentation in Kabyle and Hebrew », Nouveaux cahiers de linguistique française, 207-218.

MILLER PH. \& SAG I., (1997), «French Clitic Mouvement without Clitics or Movement », Natural Language and Linguistic Theory, 15.3, Dordrecht: Kluwer, 573-639.

RIEGEL M. PELLAT J.CH. \& RIOUL R. (1994), Grammaire méthodique du français, Paris, PUF.

SYMPOSIUMS INTERNATIONAUX INTERFACES DISCOURS-PROSODIE $(2005,2007,2009)$ : IDP05, Aix-en-Provence, http://aune.lpl.univ-aix.fr/ prodige/idp05/idp05_en.htm ; IDP07, Genève, http://idp07.unige.ch/ ; IDP09, Paris, http://makino.linguist.jussieu.fr/idp09/.

TESNIERE L. (1959), Eléments de syntaxe structurale, Klincksieck, Paris.

\section{Annexe : analyse intono-syntaxique de l'extrait de 3 minutes}

\section{Syntaxe}

// : segmentation en unités illocutoires : fin d'UI ;

$<$ : segmentation en composantes illocutoires $(\mathrm{CI})$ : fin de pré-noyau ;

$>$ : segmentation en composantes illocutoires (CI) : début d'un post-noyau ;

"..." : UI associées (marqueurs de discours (ben, hein, tu vois, quoi, ...) ;

$\wedge$ : introducteur d'UI et marqueur d'entassements ;

(...) : insertion. Pour une UI parenthétique : (..//);

...// : UI enchâssée (discours rapportés ; 
+ : continuation de l'unité rectionnelle (UR ;

$=:$ parallélisme entre deux UI.

\section{Prosodie}

.... : groupe accentuel $(\mathrm{GA})$;

PI $n+$ retour à la ligne : paquet intonatif (PI) ;

$\mathrm{P} n+$ case du tableau : période ;

Syllabe soulignée en gras : réajustement de la segmentation prosodique après éclairage de la syntaxe

\begin{tabular}{|c|c|c|c|}
\hline 离 & : & 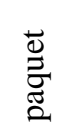 & \\
\hline \multirow[t]{3}{*}{ A.M. } & $\mathrm{P} 1$ & $\begin{array}{l}\text { PI1 } \\
\text { PI2 } \\
\text { PI3 }\end{array}$ & 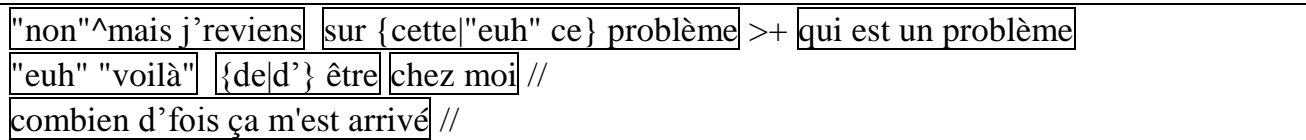 \\
\hline & $\mathrm{P} 2$ & $\begin{array}{l}\text { PI1 } \\
\text { PI2 } \\
\text { PI3 } \\
\text { PI4 } \\
\text { PI5 } \\
\text { PI6 } \\
\text { PI7 }\end{array}$ & $\begin{array}{l}\text { "bon ben" là < tu vas boulevard Voltaire // } \\
\text { c'est pas loin } / / \\
\text { "euh" \{tu|tulj'\}y vais à pieds } / / \\
\text { je suis chez moi // ie m'conditionne dans mon appartement en m'disant } \\
\text { [ i'y vais à pieds //] // } \\
\text { moi < ma voiture elle est garée dans la rue // } \\
\text { j’ai un stationnement résident // }\end{array}$ \\
\hline & P3 & $\begin{array}{l}\text { PI1 } \\
\text { PI2 } \\
\text { PI3 } \\
\text { PI4 } \\
\text { PI5 } \\
\text { PI6 }\end{array}$ & $\begin{array}{l}\text { je passe devant // } \\
\text { je n' peux pas m'empêcher \{d'ouvrir } \\
\text { "euh" la porte I de monter dedans||et d'aller "euh" à } \\
\text { "euh" "voilà" cinq minutes en voiture \} // } \\
\text { 'cqui me mettrait peut-être } \\
\text { "euh" un petit quart d'heure à pieds // } \\
\end{array}$ \\
\hline S.B. & & & $\begin{array}{l}\text { ^mais }\{\text { pour l'instant (c'est une vraie question "hein"//) } \mid \text { pour l'instant\} }<\text { c'est quand } \\
\text { même (en termes de temps) rentable aussi d'prendre la voiture // c'est \& ça va plus vite } \\
\text { qu'à pieds // }\end{array}$ \\
\hline \multirow[t]{4}{*}{ A.M. } & $\mathrm{P} 4$ & $\begin{array}{l}\text { PI1 } \\
\text { PI2 }\end{array}$ & $\begin{array}{l}\text { P4. } \wedge \text { donc au dernier moment } \\
\text { je prends ma voiture } / /\end{array}$ \\
\hline & P5 & $\begin{array}{l}\text { PI1 } \\
\text { PI2 } \\
\text { PI3 }\end{array}$ & $\begin{array}{l}\text { sur le coup } \\
\text { j' me dis } \\
{[\text { j' vais mettre cinq minutes //] // }}\end{array}$ \\
\hline & P6 & $\begin{array}{l}\text { PI1 } \\
\text { PI2 } \\
\text { PI3 } \\
\text { PI4 } \\
\text { PI5 } \\
\text { PI6 }\end{array}$ & $\begin{array}{l}\text { mais } \\
\text { le temps }\{\text { de m' garer } \mid \\
\text { de tourner | } \\
\text { de faire des ronds } \\
\text { pour pas mal me garer et tout }\}< \\
\text { je sais qu' je suis perdante } / / \\
\end{array}$ \\
\hline & & & je l'sais $>$ qu'j'suis perdante // \\
\hline S.B. & & & en fait $<$ vous êtes maintenant perdante $/ / \wedge$ mais quand même $<$ y a le \& // \\
\hline A.M. & & & y a le $\left(* p f f^{*}\right) / /$ \\
\hline & P7 & $\begin{array}{l}\text { PI1 } \\
\text { PI2 } \\
\text { PI3 } \\
\text { PI4 } \\
\text { PI5 }\end{array}$ & 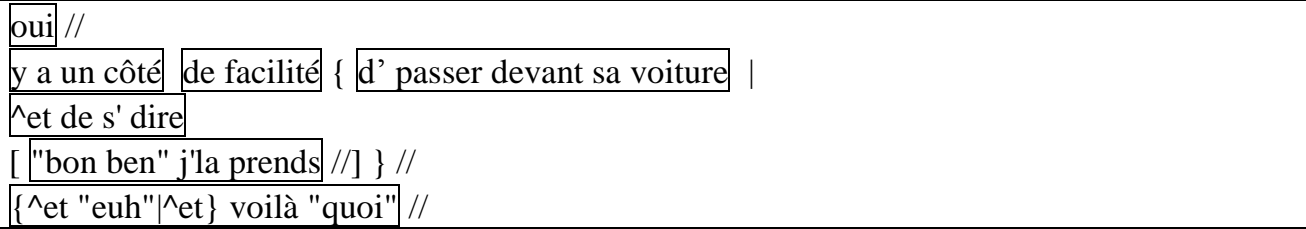 \\
\hline & P8 & $\begin{array}{l}\text { PI1 } \\
\text { PI2 }\end{array}$ & $\begin{array}{l}\text { à mon avis < c'est pas très malin } / / \\
\text { faudrait que j' \& } \\
\text { c'est une habitude (en tous cas) qu' j'aimerais changer // }\end{array}$ \\
\hline
\end{tabular}




\begin{tabular}{|c|c|c|c|}
\hline$S . B$ & & & $\begin{array}{l}\text { ^et alors < comment vous vivez \{les|la|la\} politique de la ville de Paris //+ qui est quand } \\
\text { même une chasse aux voitures // }\end{array}$ \\
\hline A.M. & P9 & PI1 & "ah ben" $\{$ jelje $\}$ suis pour $/ /=$ \\
\hline S.B. & & & vous êtes pour $/ /=$ \\
\hline \multirow[t]{23}{*}{ A.M. } & $\mathrm{P} 10$ & PI1 & je suis pour // \\
\hline & P11 & PI1 & en rentrant \\
\hline & & PI2 & dans un processus \\
\hline & & PI3 & que j'n'aime \\
\hline & & PI4 & pas trop vis-à-vis d' moi // \\
\hline & & PI5 & c'est-à-dire que "euh" \{il faut \\
\hline & & PI6 & $\begin{array}{c}\text { me mettre des li- } / \text { il faut quand même me mettre des limites }\} \text { pour m'empêcher } \\
\text { de prendre la voiture // alors que ça devrait venir de moi // }\end{array}$ \\
\hline & $\mathrm{P} 12$ & $\begin{array}{l}\text { PI1 } \\
\text { PI2 }\end{array}$ & $\begin{array}{l}\text { "c'est bon" j'aurais pas toujours cette pensée sur d'autres domaines // } \\
\text { mais sur celle-là < ouil // }\end{array}$ \\
\hline & P13 & PI1 & voilà // \\
\hline & & PI2 & par contre < j'ai essayé le vélib \{deux|trois\} fois en mettant ma carte bleue $/ /$ \\
\hline & & PI3 & 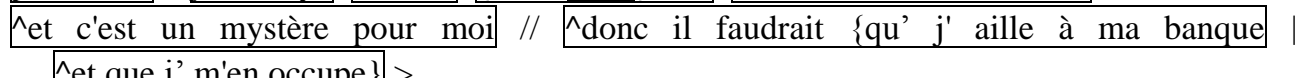 \\
\hline & & PI4 & quand même // \\
\hline & P14 & PI1 & $\mid "$ "euh" ^puisque ma caution $<\{$ \{quand on met sa carte bleue $\mid$ qu'on a pas d' carte d'abonné $\}<$ \\
\hline & & PI2 & ma caution $<$ y a écrit [ caution $<$ \\
\hline & & PI3 & cent cinquante euros //] // \\
\hline & & PI4 & j'valide // \\
\hline & & PI5 & ^et elle m'est refusée //+ \\
\hline & P15 & PI1 & même \\
\hline & & PI2 & \\
\hline & & PI3 & "euh" \{y a quand même $\mid$ y a $\}$ \{des|des $\}$ sous sur le compte // \\
\hline & & PI4 & $\wedge$ donc "euh" voilà // \\
\hline & P16 & $\begin{array}{l}\text { PI1 } \\
\text { PI2 }\end{array}$ & \begin{tabular}{|l|} 
donc je n'ai pas eu droit \\
encore //
\end{tabular} \\
\hline & & & j’ai pas réussi à accéder// \\
\hline S.B. & & & "euh" "oui" ^mais vous avez essayé // le deux-roues <ça vous déplairait pas // \\
\hline \multirow[t]{6}{*}{ A.M. } & P17 & PI1 & le vélo $<$ \\
\hline & & PI2 & si > pour essayer une fois // \\
\hline & & PI3 & "non" ^mais j' crois que je suis \& comme j’ai jamais fait quasi d' vélo d’ ma vie $<$ \\
\hline & & PI4 & "euh" \{en tant que vraie parisienne $\mid$ ^et en étant très peu partie à la campagne $\}<$ j' suis \\
\hline & & PI5 & \{pas|pas\} très à l'aise // \\
\hline & & PI6 & ^mais pour faire les petites distances $<\& / /$ \\
\hline S.B. & & & vos filles ont \{quel âge $\mid\} / /+$ \\
\hline A.M. & & & $\wedge$ alors $\{\mid\{\{$ dix-sept $\mid \wedge$ et demi $\} \mid \wedge$ et quinze ans $\}\} / /$ \\
\hline \multirow[t]{2}{*}{ S.B. } & & & ^et c'est aussi des accros d' la voiture // \\
\hline & $\mathrm{P} 18$ & PI1 & "euh" non // \\
\hline \multirow[t]{8}{*}{ A.M. } & P19 & PI1 & elles marchent $/ /=$ \\
\hline & & PI2 & elles s' \& "non non" elles ont tendance à \& //= "non non" elles adorent marcher // \\
\hline & $\mathrm{P} 20$ & PI1 & ^ppuisque moi < "voilà" j' peux m' dire ^mais j' viens t' chercher // \\
\hline & & PI2 & tu finis tard // \\
\hline & & PI3 & tu seras \& voilà // // \\
\hline & $\mathrm{P} 21$ & PI1 & non non non // \\
\hline & & PI2 & laisse-moi marcher $/ /=$ laisse-moi marcher $/ / \wedge^{\wedge}$ donc "euh" non non $/ /$ \\
\hline & $\mathrm{P} 22$ & PI1 & $\begin{array}{l}\text { pareil qu'elles marchent } / /=\text { elles prennent pas l' métro } \\
\text { //= elles prennent pas l' métro } / /=\text { elles y vont à pieds } / /\end{array}$ \\
\hline
\end{tabular}


[Texte] 